\title{
A Facile One-Pot Benzylation of Sodium Enolates Using Trifluoromethanesulfonic Anhydride and Diphenyl Sulfoxide
}

\author{
Tomofumi TAKuwA, ${ }^{a}$ Tomofumi Minowa, ${ }^{a}$ Hidehiko FusisawA, ${ }^{a}$ and Teruaki MukaiYamA ${ }^{*, a, b}$ \\ ${ }^{a}$ Center for Basic Research, The Kitasato Institute; 6-15-5 (TCI) Toshima, Kita-ku, Tokyo 114-0003, Japan: and \\ ${ }^{b}$ Kitasato Institute for Life Science, Kitasato University; 5-9-1 Shirokane, Minato-ku, Tokyo 108-8641, Japan. \\ Received October 1, 2004; accepted March 4, 2005; published online March 10, 2005
}

\begin{abstract}
A facile one-pot $\boldsymbol{C}$-benzylation of various sodium enolates derived from methyl malonate, $\beta$-ketoesters, a $\beta$ cyanoester, a $\beta$-cyanosulfone, ketones and a carboxylic ester is reported. Reaction of alkoxydiphenylsulfonium salts formed by treating various benzyl alcohols with diphenyl sulfide bis(trifluoromethanesulfonate) (derived from trifluoromethanesulfonic anhydride and diphenyl sulfoxide) proceeded smoothly, and the corresponding $C$ benzylated products were afforded in good to high yields.
\end{abstract}

Key words $\quad C$-benzylation; diphenyl sulfoxide; trifluoromethanesulfonic anhydride

Alkylation of metal enolates is one of the most fundamental and frequently used reactions in organic synthesis. This reaction is generally accomplished by treating various metal enolates with alkylating reagents such as alkyl halides or alkyl sulfonates. Such reactions of metal enolates derived from ketones, ${ }^{1)}$ esters, ${ }^{2)}$ and $\beta$-keto esters ${ }^{3)}$ with alkyl halides afford a mixture of mono-, di- and poly-alkylated products, even when one equivalent each of base and alkylating reagent are used. For example, $O$-monobenzylated, $C$ monobenzylated and $C$-dibenzylated products $(5: 45: 50)$ were obtained by benzylation of ethyl acetate with benzyl bromide in dimethyl sulfoxide. ${ }^{4)}$

$C$-Alkylation of active methylene compounds with alcohols has recently been accomplished in a redox reaction system by Mitsunobu-type reactions, ${ }^{5)}$ and Tsunoda and coworkers $^{(6)}$ have also reported efficient methods for alkylation of active methylene compounds. However, with benzyl alcohol, it is reported that the concomitant double alkylation and/or an ether forming-reaction takes place. ${ }^{7)}$ Thus, it is important to develop an efficient approach to this type of reaction, since $C$-monobenzylation of enolates is an important area of synthetic organic chemistry.

The ratio of $C$ - or $O$-alkylation strongly depends on the nature of the alkylating reagent. Alkoxydiphenyl sulfonium species are known as efficient alkylating reagents in reactions with carbon nucleophiles. Similarly, sulfonium salts derived from dialkylsulfoxides and trifluoromethanesulfonic anhydride have been utilized previously in Swern-type oxidation, ${ }^{8)}$ sulfilimine synthesis, ${ }^{9)}$ and glycosylation reactions. ${ }^{10)}$ Here, we describe a facile one-pot $C$-benzylation using trifluoromethanesulfonic anhydride and diphenyl sulfoxide. ${ }^{11)}$

\section{Results and Discussion}

Generation of an alkoxy sulfonium intermediate was first attempted using the bromodiphenyl sulfonium salt derived from diphenyl sulfide and bromine (Chart 1); that is, $1.0 \mathrm{eq}$ of bromine was added to diphenyl sulfoxide in dichloromethane at $-78^{\circ} \mathrm{C}$. After stirring the mixture for $10 \mathrm{~min}$, 4-methoxybenzyl alcohol and freshly-prepared sodium enolate of dimethyl malonate were continuously added, followed by slow warming to room temperature. However, the desired product was not obtained. We have previously reported that diphenyl sulfide bis(trifluoromethanesulfonate) reacts with styrene to generate the corresponding ac- tive sulfonium intermediate, which in turn reacts with amines to afford aziridines or $\beta, \gamma$-unsaturated amines. ${ }^{12,13)}$ Therefore, formation of an alkoxy diphenyl sulfonium intermediate was anticipated upon treatment of an alcohol with diphenyl sulfide bis(trifluoromethanesulfonate). Initially, reaction of 4methoxybenzyl alcohol with sodium dimethyl malonate was studied as a model system; that is, the diphenyl alkoxy sulfonium intermediate was generated by adding 1.2 eq of 4methoxybenzyl alcohol to a mixture of trifluoromethanesulfonic anhydride and diphenyl sulfoxide in tetrahydrofuran (THF) at $-78^{\circ} \mathrm{C}$. After stirring the mixture for $30 \mathrm{~min}$, 3.0 eq of freshly-prepared sodium enolate of dimethyl malonate was continuously added and then the reaction mixture was slowly warmed to room temperature. After aqueous work up, dimethyl (4-methoxybenzyl)malonate was obtained in $35 \%$ yield (Chart 2 ).

In order to improve the efficiency of the reaction, optimization of the reaction conditions was attempted. First, the effects of various solvents were examined (Table 1). When solvents such as $\mathrm{MeCN}, \mathrm{CH}_{2} \mathrm{Cl}_{2}$, hexane and dimethylformamide (DMF) were used, the desired product was not de-

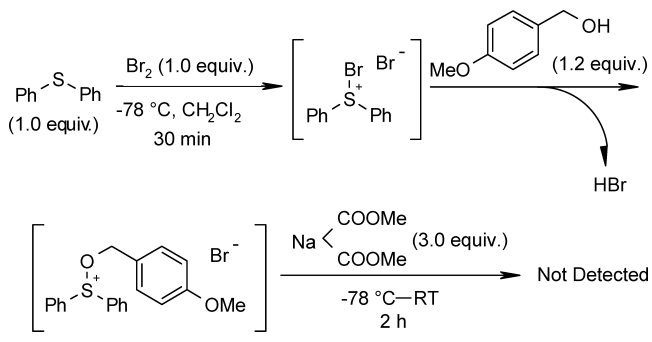

Chart 1
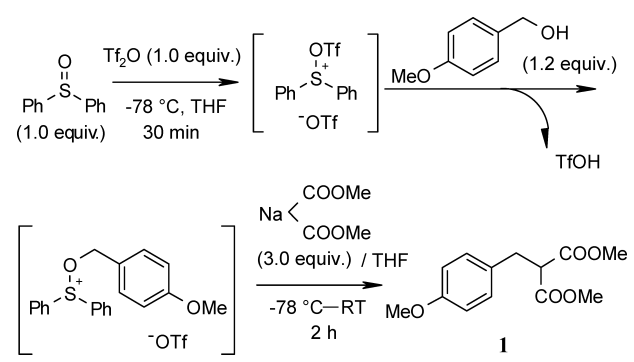

Chart 2 
Table 1. Effects of Various Solvents

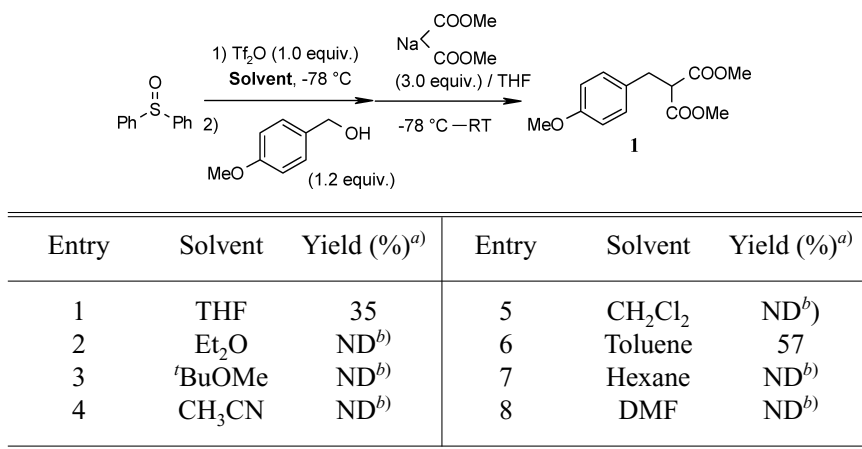

a) Isolated yields. b) Desired product was not detected.

Table 2. Effect of Bases as a Scavenger in Benzylation

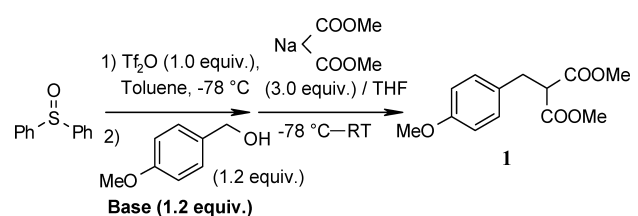

\begin{tabular}{|c|c|c|c|c|c|}
\hline Entry & Base & $\begin{array}{l}\text { Yield } \\
(\%)^{a)}\end{array}$ & Entry & Base & $\begin{array}{l}\text { Yield } \\
(\%)^{a)}\end{array}$ \\
\hline 1 & None & 57 & 6 & $\mathrm{DBU}^{b)}$ & Trace \\
\hline 2 & $\mathrm{CsF}$ & 54 & 7 & $N, N$-Dimethylaniline & Trace \\
\hline 3 & $\mathrm{Cs}_{2} \mathrm{CO}_{3}$ & 58 & 8 & Pyridine & 77 \\
\hline 4 & $\mathrm{~K}_{2} \mathrm{CO}_{3}$ & 65 & 9 & 2,6-Lutidine & 53 \\
\hline 5 & Triethylamine & Trace & 10 & Proton Sponge ${ }^{\circledR c)}$ & 97 \\
\hline
\end{tabular}

a) Isolated yields. b) 1,8-Diazabicyclo[5.4.0]undec-7-ene. c) in

tected. Similar results were obtained when ethereal solvents such as $\mathrm{Et}_{2} \mathrm{O}$ and ${ }^{t} \mathrm{BuOMe}$ were used. In contrast, the reaction proceeded smoothly when toluene was used as solvent, and the desired product was obtained in 57\% yield (Entry 6). During this reaction, $\mathrm{TfOH}$ was generated as the reaction of the diphenyl sulfonium intermediate and 4-methoxybenzyl alcohol progressed. Thus, the reaction was examined in the presence of various bases, in order to capture $\mathrm{TfOH}$ as it was formed. The effects of these bases are shown in Table 2 . When solid bases such as cesium fluoride, cesium carbonate and potassium carbonate were used, the results were similar to the reaction carried out in the absence of base. Therefore, organic bases such as $\mathrm{Et}_{3} \mathrm{~N}, 1,8$-diazabicyclo[5.4.0]undec-7ene (DBU), and $\mathrm{PhNMe}_{2}$ were chosen so that the reaction could be performed in a homogeneous system. However, only a trace amount of the desired product was detected with these bases. This indicates that preferential nucleophilic attack by the amine on diphenyl sulfide bis(trifluoromethanesulfonate) occurred faster than formation of the alkoxy diphenyl sulfonium intermediate, since these amine bases are more nucleophilic than alcohols. In order to reduce this effect, the reaction was performed using weaker amine nucleophiles, such as pyridine and 2,6-lutidine. With pyridine, the desired product was obtained in $77 \%$ yield, while a yield similar to that in the absence of bases was obtained with 2,6litidine. Furthermore, the reaction proceeded smoothly to afford the $C$-benzylated product in an almost quantitative yield when proton sponge ${ }^{\circledR}$ was used as a base, although the rea-
Table 3. The Amount of Sodium Enolate

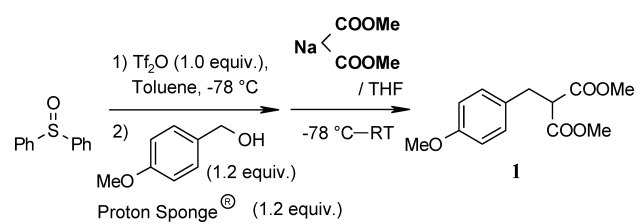

\begin{tabular}{cccc}
\hline \hline Entry & Nucleophile & Equivalent & Yield (\%) ${ }^{a)}$ \\
\hline 1 & & 1.5 & 25 \\
2 & $\mathrm{Na}<$ COOMe & 2.0 & 68 \\
3 & & 3.0 & 97 \\
\hline
\end{tabular}

a) Isolated yields.

sons for the particular effect of proton sponge ${ }^{\circledR}$ remain unclear.

Next, the appropriate amount of nucleophile was examined (Table 3). Yields were lowered by decreasing the amount of nucleophile, and at least $3.0 \mathrm{eq}$ of nucleophile were necessary for preparation of the desired product in high yield. This result indicates that the nucleophiles are protonated by TfOH trapped with the base and by excess benzyl alcohol. In all cases, dibenzylated and $O$-benzylated products were not detected under these conditions.

The effects of alcohols and nucleophiles were then examined under the optimized reaction conditions. The effects of benzyl alcohol substituents were examined using the sodium enolate of dimethyl malonate as a model nucleophile (Table 4). All reactions were performed using the same reaction time, and it was found that various benzyl alcohols can be effectively employed in the reaction. For benzyl alcohols with electron-donating groups, the desired products were obtained in high yields (Entries 3-6). Furthermore, the corresponding $C$-benzylated products were obtained in good to high yields when benzyl alcohols with electron-withdrawing groups were used (Entries 8-12). In contrast, the desired product was not detected for a secondary benzyl alcohol such as 1-phenylethanol (Entry 13).

It was noted that the desired product was not detected when the reaction was carried out in the absence of diphenyl sulfoxide (Table 4, Entry 2). This indicates that the reaction does not proceed via nucleophilic substitution of benzyl trifluoromethanesulfonate (generated from benzyl alcohol and trifluoromethanesulfonic anhydride) by the nucleophile.

Several nucleophiles were further examined (Table 5) and the desired products were obtained in excellent yields with $\beta$-keto esters (Entries 2, 3). When the sodium enolate of methyl cyanoacetate was used as a nucleophile, the corresponding product was obtained in good yield with 4.0 eq of nucleophile (Entry 4), and the sodium salt of (phenylsulfonyl)acetonitrile also worked effectively in the reaction (Entry 5). Similarly, the reaction proceeded smoothly with sodium enolates derived from various ketones, using 4.06.0 eq of nucleophile (Entries 6-9). Furthermore, a sodium enolate derived from a carboxylic ester also served as a nucleophile in the reaction, again using 4.0 eq of nucleophile (Entry 10).

Next, the effect of the counter cation in the metal enolates was investigated (Table 6). With lithium or potassium enolates generated from acetophenone, the desired product was 
Table 4. Various Benzyl Alcohol Derivatives in Benzylation Reactions

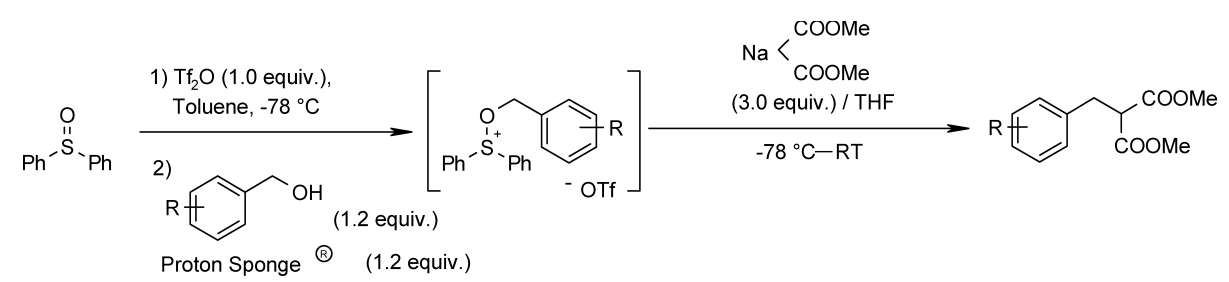

\begin{tabular}{|c|c|c|c|c|c|c|c|}
\hline Entry & Alcohol & Product & Yield $(\%)^{a)}$ & Entry & Alcohol & Product & Yield $(\%)^{a)}$ \\
\hline 1 & & 2 & 94 & 8 & & 7 & 74 \\
\hline $2^{b)}$ & & - & $\mathrm{ND}^{c)}$ & 9 & & 8 & 88 \\
\hline 3 & & 1 & 97 & 10 & & 9 & 74 \\
\hline 5 & & 4 & 85 & 11 & & 10 & 84 \\
\hline 6 & & 5 & 85 & 12 & & 11 & 76 \\
\hline 7 & & 6 & 83 & 13 & & - & $\mathrm{ND}^{c)}$ \\
\hline
\end{tabular}

a) Isolated yields. b) The reaction was carried out without diphenyl sulfoxide. c) Desired product was not detected.

Table 5. Investigation of Other Sodium Enolates

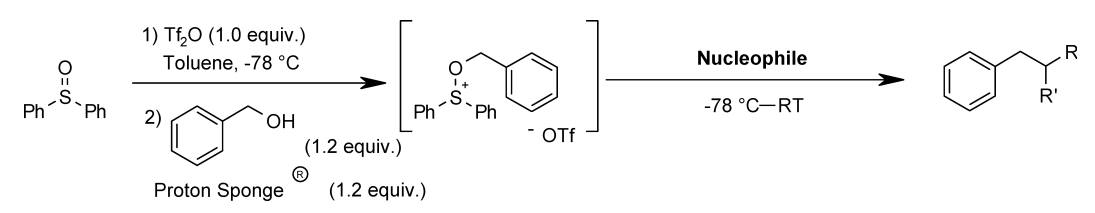

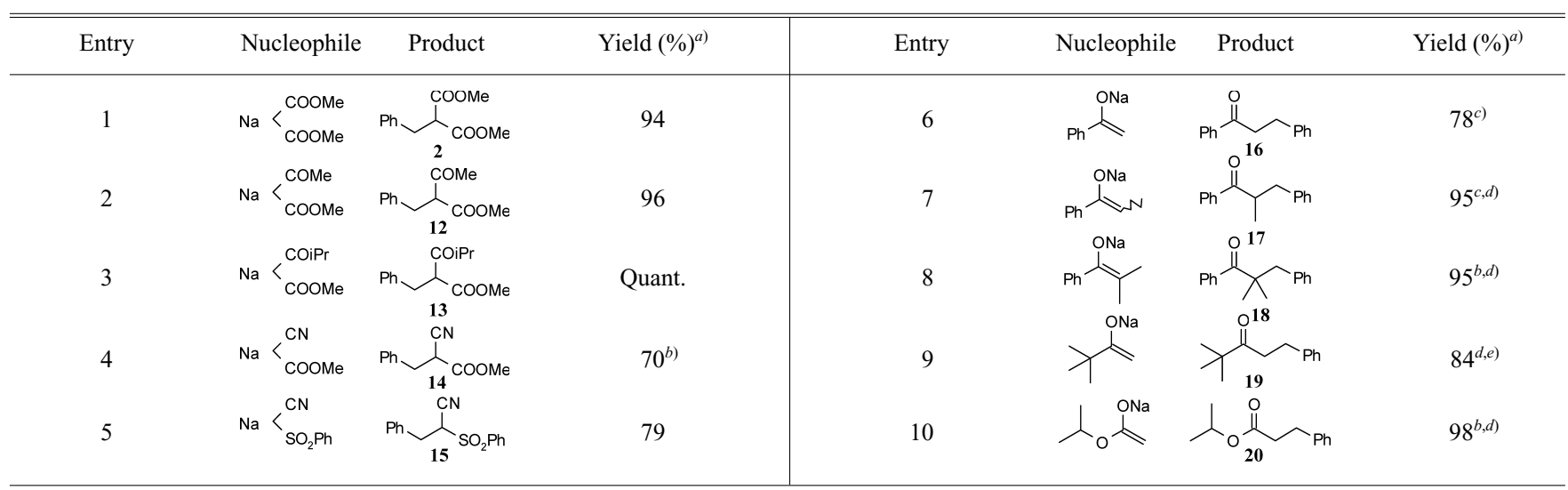
used.

a) Isolated yields. b) 4.0 eq of nucleophiles were used. c) 5.0 eq of nucleophiles were used. $\quad$ d) Yields were determined by ${ }^{1} \mathrm{H}-\mathrm{NMR}$. e) 6.0 eq of nucleophiles were

obtained in $38 \%$ or $30 \%$ yields, respectively. In contrast, the reaction proceeded smoothly and afforded the desired product in good yield in the presence of a sodium enolate nucleophile.

Finally, the reaction was attempted using other alcohols that were not simple benzyl alcohols. For example, phenethyl alcohol was used for alkylation of the sodium enolate generated from isopropyl acetate, but the corresponding product was not detected. To increase the reactivity of the alkoxy sulfonium intermediate, alkoxy dialkyl sulfonium salts of phenethyl alcohol and di-p-nitrophenyl or di-tert-butylphenyl sulfoxide were used, but the reaction did not proceed at all (Chart 3). This suggests that the reaction is limited to the primary benzyl alcohol at present (Table 4, Entry 13). The reaction mechanism is assumed to be that shown in Chart 4. Initially, diphenyl sulfoxide is activated by trifluoromethanesulfonic anhydride to form a sulfonium complex A. Reaction of the benzyl alcohol with A in situ affords an active oxosulfonium trifluoromethanesulfonate $\mathrm{B}$, which in turn reacts with a nucleophile to give the corresponding $C$-benzylated product. 
Table 6. Other Metal Enolate

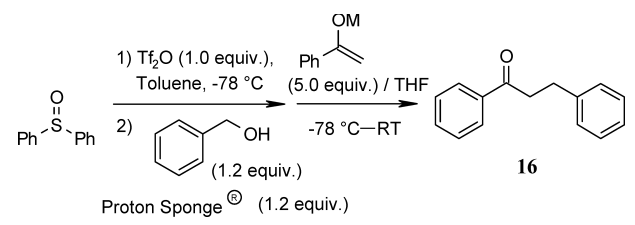

\begin{tabular}{ccc}
\hline \hline Entry & Metal & Yield (\%) ${ }^{a)}$ \\
\hline 1 & $\mathrm{Li}$ & 38 \\
2 & $\mathrm{Na}$ & 78 \\
3 & $\mathrm{~K}$ & 30 \\
\hline
\end{tabular}

a) Isolated yields.
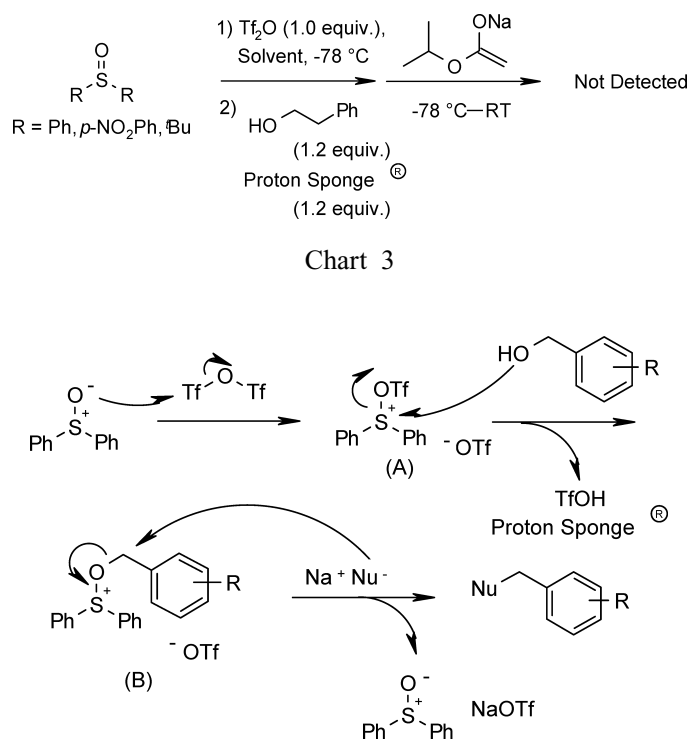

Chart 4

\section{Conclusion}

A new and efficient one-pot $C$-benzylation reaction of various sodium enolates was established using new benzylating reagents generated in situ from diphenyl sulfoxide, trifluoromethanesulfonic anhydride and benzyl alcohols. Further study of this type of alkylation reaction is currently in progress.

\section{Experimental}

All melting points were determined on a Yanagimoto micro melting point apparatus (Yanaco MP-S3) and were uncorrected. Infrared (IR) spectra were recorded on a Horiba FT 300 FT-IR spectrometer. ${ }^{1} \mathrm{H}-\mathrm{NMR}$ spectra were recorded on a JEOL EX270 $(270 \mathrm{MHz})$ spectrometer; chemical shifts $(\delta)$ are reported in parts per million relative to tetramethylsilane. Splitting patterns are designed as s, singlet; $d$, doublet; $t$, triplet; q, quartet; $\mathrm{m}$, multiplet; br, broad. ${ }^{13} \mathrm{C}$-NMR spectra were recorded on a JEOL EX270 $(68 \mathrm{MHz})$ spectrometer with complete proton decoupling. Chemical shifts are reported in parts per million relative to tetramethylsilane with the solvent resonance as the internal standard $\left(\mathrm{CDCl}_{3} ; \delta 77.0 \mathrm{ppm}\right)$. High-resolution mass spectra (HR-MS) were recorded on a JEOL-700T mass spectrometer. Elemental analyses were performed on vario EL III (elementar), DX-500 (DIONEX) and UV2200 (SHIMADZU). Analytical TLC was performed on Merck precoated TLC plates (silica gel $60 \mathrm{GF} 254,0.25 \mathrm{~mm}$ ). Silica-gel column chromatography was carried out on Merck silica gel $60(0.063-0.200 \mathrm{~mm})$. Preparative thin-layer chromatography (PTLC) was carried out on silica-gel Wacogel B-5F. Solvents were freshly distilled when dry solvents were needed. Other reagents were purchased from Tokyo Kasei Kogyo, Kanto Chemical, Aldrich Chemical or Merck, and were used after purification by distillation or recrystallization.

Typical Experimental Procedure for Benzylation of Enolates The ex- perimental procedure (Table 4, Entry 3 ) is described: to a stirred solution of diphenyl sulfoxide $(0.20 \mathrm{mmol})$ in toluene $(1.0 \mathrm{ml})$ under an argon atmosphere was added a trifluoromethanesulfonic anhydride $(0.20 \mathrm{mmol})$ at $-78^{\circ} \mathrm{C}$. After stirring for $30 \mathrm{~min}$ a solution of 4-methoxybenzyl alcohol $(0.24 \mathrm{mmol})$ and proton sponge ${ }^{\mathbb{B}}(0.24 \mathrm{mmol})$ in toluene $(0.8 \mathrm{ml})$ was added, and the reaction mixture was stirred for further $30 \mathrm{~min}$. Fleshly prepared sodium salt ${ }^{14)}$ of dimethyl malonate dissolved in THF $(0.4 \mathrm{M}, 1.5 \mathrm{ml})$ was added and the reaction mixture was slowly warmed up to room temperature. After stirring for $2 \mathrm{~h}$, the reaction mixture was quenched with water $(5 \mathrm{ml})$ and the aqueous layer was extracted with ethyl acetate $(30 \mathrm{ml})$. The organic layers were collected and dried $\left(\mathrm{MgSO}_{4}\right)$. After filtration and evaporation of the solvdent, the resulted residue was purified by preparative TLC to afford compound $\mathbf{1}$ in $97 \%$ yield as a colorless oil.

Dimethyl (4-Methoxybenzyl)malonate (1) ${ }^{15)}$ IR (neat) $\mathrm{cm}^{-1}: 3779$, 2360, 1743, 1249, 1218. ${ }^{1} \mathrm{H}-\mathrm{NMR}\left(270 \mathrm{MHz}, \mathrm{CDCl}_{3}\right) \delta: 3.14(2 \mathrm{H}, \mathrm{d}$, $J=7.8 \mathrm{~Hz}), 3.61(1 \mathrm{H}, \mathrm{d}, J=7.8 \mathrm{~Hz}), 3.68(6 \mathrm{H}, \mathrm{s}), 3.75(3 \mathrm{H}, \mathrm{s}), 6.79(2 \mathrm{H}, \mathrm{d}$, $J=8.7 \mathrm{~Hz}), 7.19(2 \mathrm{H}, \mathrm{d}, J=8.7 \mathrm{~Hz}) .{ }^{13} \mathrm{C}-\mathrm{NMR}\left(68 \mathrm{MHz}, \mathrm{CDCl}_{3}\right) \delta: 34.0$, $52.6(\times 2), 53.9,55.2,113.8,129.6(\times 2), 129.7(\times 2), 158.2,169.1(\times 2)$.

Dimethyl Benzylmalonate (2) ${ }^{16)}$ IR (neat) $\mathrm{cm}^{-1}: 2360,2337,1743$, 1442, 1234. ${ }^{1} \mathrm{H}-\mathrm{NMR}\left(270 \mathrm{MHz}, \mathrm{CDCl}_{3}\right) \delta: 3.21(2 \mathrm{H}, J=7.9 \mathrm{~Hz}, \mathrm{~d}), 3.64-$ $3.67(1 \mathrm{H}, \mathrm{m}), 3.68(6 \mathrm{H}, \mathrm{s}), 7.16-7.29(5 \mathrm{H}, \mathrm{m}) .{ }^{13} \mathrm{C}-\mathrm{NMR}\left(68 \mathrm{MHz}, \mathrm{CDCl}_{3}\right)$ $\delta: 34.8,52.6(\times 2), 53.6,126.7,128.4(\times 2), 128.6(\times 2), 137.6,169.0(\times 2)$.

Dimethyl (3-Methoxybenzyl)malonate (3) ${ }^{17)}$ IR (neat) $\mathrm{cm}^{-1}: 3586$, 2337, 1743, 1265. ${ }^{1} \mathrm{H}-\mathrm{NMR}\left(270 \mathrm{MHz}, \mathrm{CDCl}_{3}\right) \delta: 3.17(2 \mathrm{H}, \mathrm{d}, J=7.8 \mathrm{~Hz})$, $3.62-3.65(1 \mathrm{H}, \mathrm{m}), 3.67(6 \mathrm{H}, \mathrm{s}), 3.75(3 \mathrm{H}, \mathrm{s}), 6.71-6.75(3 \mathrm{H}, \mathrm{m}), 7.16$ $(1 \mathrm{H}, \mathrm{t}, J=7.8 \mathrm{~Hz}) .{ }^{13} \mathrm{C}-\mathrm{NMR}\left(68 \mathrm{MHz}, \mathrm{CDCl}_{3}\right) \delta: 34.8,52.6(\times 2), 53.5$, $55.1,112.1,114.3,120.9,129.4,139.1,159.5,169.0(\times 2)$.

Dimethyl (4-Methylbenzyl)malonate (4) ${ }^{18)}$ IR (neat) $\mathrm{cm}^{-1}: 2360$, 1743, 1442, 1226, 809. ${ }^{1} \mathrm{H}-\mathrm{NMR}\left(270 \mathrm{MHz}, \mathrm{CDCl}_{3}\right) \delta: 2.29(3 \mathrm{H}, \mathrm{s}), 3.16$ $(2 \mathrm{H}, \mathrm{d}, J=7.8 \mathrm{~Hz}), 3.63(1 \mathrm{H}, \mathrm{t}, J=7.9 \mathrm{~Hz}), 3.68(6 \mathrm{H}, \mathrm{s}), 7.06(4 \mathrm{H}, \mathrm{s}) .{ }^{13} \mathrm{C}-$ NMR $\left(68 \mathrm{MHz}, \mathrm{CDCl}_{3}\right) \delta: 21.1,34.4,52.6(\times 2), 53.7,128.5(\times 2), 129.1$ $(\times 2), 130.7,136.2,169.1(\times 2)$

Dimethyl (4-Isopropylbenzyl)malonate (5) ${ }^{19)}$ IR (neat) $\mathrm{cm}^{-1}: 2962$, $2337,1743,1434,823 .{ }^{1} \mathrm{H}-\mathrm{NMR}\left(270 \mathrm{MHz}, \mathrm{CDCl}_{3}\right) \delta: 1.21(2 \mathrm{H}, \mathrm{d}$, $J=6.9 \mathrm{~Hz}), 2.80-2.90(1 \mathrm{H}, \mathrm{m}), 3.18(2 \mathrm{H}, \mathrm{d}, J=7.8 \mathrm{~Hz}), 3.62-3.65(1 \mathrm{H}$, $\mathrm{m}), 3.68(6 \mathrm{H}, \mathrm{s}), 7.10(4 \mathrm{H}, \mathrm{m}) .{ }^{13} \mathrm{C}-\mathrm{NMR}\left(68 \mathrm{MHz}, \mathrm{CDCl}_{3}\right) \delta: 24.0(\times 2)$, $33.7,34.4,52.5(\times 2), 53.7,126.5,(\times 2), 128.5(\times 2), 134.9,147.2,169.1$ $(\times 2)$.

Dimethyl (1-Naphthylmethyl)malonate (6) IR (neat) $\mathrm{cm}^{-1}: 3070$, 2337, 1743, 1442, 786. ${ }^{1} \mathrm{H}-\mathrm{NMR}\left(270 \mathrm{MHz}, \mathrm{CDCl}_{3}\right) \delta: 3.68(6 \mathrm{H}, \mathrm{s}), 3.73$ $(2 \mathrm{H}, \mathrm{d}, J=5.0 \mathrm{~Hz}), 3.87(1 \mathrm{H}, \mathrm{t}, J=7.5 \mathrm{~Hz}), 7.36(2 \mathrm{H}, \mathrm{m}), 7.50(2 \mathrm{H}, \mathrm{m}), 7.73$ $(1 \mathrm{H}, \mathrm{dd}, J=6.3,3.3 \mathrm{~Hz}), 7.85(1 \mathrm{H}, \mathrm{d}, J=7.6 \mathrm{~Hz}), 8.01(1 \mathrm{H}, \mathrm{d}, J=8.2 \mathrm{~Hz})$. ${ }^{13} \mathrm{C}-\mathrm{NMR}\left(68 \mathrm{MHz}, \mathrm{CDCl}_{3}\right) \delta: 32.0,52.59,52.61(\times 2), 123.0,125.3,125.6$, $126.2,127.0,127.6,128.9,131.3,133.5,133.8,169.2(\times 2)$. Anal. Calcd for $\mathrm{C}_{16} \mathrm{H}_{16} \mathrm{O}_{4}: \mathrm{C}, 70.57 ; \mathrm{H}, 5.92$. Found: $\mathrm{C}, 70.61 ; \mathrm{H}, 5.88$.

Dimethyl (4-Nitrobenzyl)malonate (7) ${ }^{20)}$ IR (neat) $\mathrm{cm}^{-1}: 3370,2946$, $1743,1342 .{ }^{1} \mathrm{H}-\mathrm{NMR}\left(270 \mathrm{MHz}, \mathrm{CDCl}_{3}\right) \delta: 3.20(2 \mathrm{H}, \mathrm{d}, J=7.9 \mathrm{~Hz}), 3.59-$ $3.61(1 \mathrm{H}, \mathrm{m}), 3.59(6 \mathrm{H}, \mathrm{s}), 7.26(2 \mathrm{H}, \mathrm{d}, J=8.9 \mathrm{~Hz}), 8.02(2 \mathrm{H}, \mathrm{d}, J=8.9 \mathrm{~Hz})$. ${ }^{13} \mathrm{C}-\mathrm{NMR}\left(68 \mathrm{MHz}, \mathrm{CDCl}_{3}\right) \delta: 34.4,52.5,52.8(\times 2), 123.7(\times 2), 125.3$, $129.6(\times 2), 145.2,168.4(\times 2)$.

Dimethyl (4-Chlorobenzyl)malonate $(\mathbf{8})^{21)}$ IR (neat) $\mathrm{cm}^{-1}$ : 3756, 2337, 1743, 848, 817. ${ }^{1} \mathrm{H}-\mathrm{NMR}\left(270 \mathrm{MHz}, \mathrm{CDCl}_{3}\right) \delta: 3.60(2 \mathrm{H}, \mathrm{d}$, $J=7.8 \mathrm{~Hz}), 3.62(1 \mathrm{H}, \mathrm{t}, J=7.8 \mathrm{~Hz}), 3.68(6 \mathrm{H}, \mathrm{s}), 7.11(2 \mathrm{H}, \mathrm{d}, J=8.1 \mathrm{~Hz})$, $7.22(2 \mathrm{H}, \mathrm{d}, J=8.2 \mathrm{~Hz}) .{ }^{13} \mathrm{C}-\mathrm{NMR}\left(68 \mathrm{MHz}, \mathrm{CDCl}_{3}\right) \delta: 34.1,52.6(\times 2)$, $53.4,128.6(\times 2), 130.0(\times 2), 132.5,136.0,167.8(\times 2)$.

Dimethyl (3-Chlorobenzyl)malonate (9) IR (neat) $\mathrm{cm}^{-1}: 2954,2337$, $1743,1442,1025 .{ }^{1} \mathrm{H}-\mathrm{NMR}\left(270 \mathrm{MHz}, \mathrm{CDCl}_{3}\right) \delta: 3.17(2 \mathrm{H}, \mathrm{d}, J=7.8 \mathrm{~Hz})$, $3.63(1 \mathrm{H}, \mathrm{t}, J=7.8 \mathrm{~Hz}), 3.69(6 \mathrm{H}, \mathrm{s}), 7.04-7.30(4 \mathrm{H}, \mathrm{m}) .{ }^{13} \mathrm{C}-\mathrm{NMR}$ $\left(68 \mathrm{MHz}, \mathrm{CDCl}_{3}\right) \delta: 34.4,52.7(\times 2), 53.3,126.9,127.0,128.8,129.7$, 134.2, 139.6, $168.7(\times 2)$. FAB-HR-MS $m / z: 257.0585$ (Calcd for $\mathrm{C}_{12} \mathrm{H}_{14} \mathrm{ClO}_{4}:$ 257.0575).

Dimethyl (2-Chlorobenzyl)malonate (10) IR (neat) $\mathrm{cm}^{-1}: 2954,2360$, $2337,1743,1473 .{ }^{1} \mathrm{H}-\mathrm{NMR}\left(270 \mathrm{MHz}, \mathrm{CDCl}_{3}\right) \delta: 3.25(2 \mathrm{H}, \mathrm{d}, J=7.8 \mathrm{~Hz})$, $3.61(6 \mathrm{H}, \mathrm{s}), 3.77(1 \mathrm{H}, \mathrm{t}, J=7.8 \mathrm{~Hz}), 7.07-7.17(3 \mathrm{H}, \mathrm{m}), 7.24-7.27(1 \mathrm{H}$, m). ${ }^{13} \mathrm{C}$-NMR $\left(68 \mathrm{MHz}, \mathrm{CDCl}_{3}\right) \delta: 32.8,51.2,52.6(\times 2), 126.7,128.4$, 129.5, 131.2, 134.0, 135.1, $168.9(\times 2)$. FAB-HR-MS $m / z: 257.0579$ (Calcd for $\left.\mathrm{C}_{12} \mathrm{H}_{14} \mathrm{ClO}_{4}: 257.0575\right)$.

Dimethyl (2-Fluorobenzyl)malonate (11) ${ }^{22)}$ IR (neat) $\mathrm{cm}^{-1}: 3710$, 2352, $1743,1234 .{ }^{1} \mathrm{H}-\mathrm{NMR}\left(270 \mathrm{MHz}, \mathrm{CDCl}_{3}\right) \delta: 3.23(2 \mathrm{H}, \mathrm{d}, J=7.9 \mathrm{~Hz})$, $3.67(6 \mathrm{H}, \mathrm{s}), 3.73(1 \mathrm{H}, \mathrm{t}, J=7.8 \mathrm{~Hz}), 6.95-7.04(2 \mathrm{H}, \mathrm{m}), 7.14-7.24(2 \mathrm{H}$, m). ${ }^{13} \mathrm{C}-\mathrm{NMR}\left(68 \mathrm{MHz}, \mathrm{CDCl}_{3}\right) \delta: 28.6,51.8,52.6(\times 2), 115.1,115.4$, 124.0, 128.6, 128.7, 131.1, 168.9 (×2).

Methyl 2-Benzyl-3-oxobutanoate (12) ${ }^{23)}$ IR (neat) $\mathrm{cm}^{-1}: 2337,1735$, 1434, 1211. ${ }^{1} \mathrm{H}-\mathrm{NMR}\left(270 \mathrm{MHz}, \mathrm{CDCl}_{3}\right) \delta: 2.17(3 \mathrm{H}, \mathrm{s}), 3.15(2 \mathrm{H}, \mathrm{d}$, 
$J=7.7 \mathrm{~Hz}), 3.68(3 \mathrm{H}, \mathrm{s}), 3.79(1 \mathrm{H}, \mathrm{t}, J=7.7 \mathrm{~Hz}), 7.15-7.30(5 \mathrm{H}, \mathrm{m}) \cdot{ }^{13} \mathrm{C}-$ NMR $\left(68 \mathrm{MHz}, \mathrm{CDCl}_{3}\right) \delta: 29.8,34.0,52.5,61.1,111.2,126.6(\times 2), 128.6$ $(\times 2), 137.9,169.3,202.2$.

Methyl 2-Benzyl-4-methyl-3-oxopentanoate (13) IR (neat) $\mathrm{cm}^{-1}$ : 2954, 2329, 1743, 1712. ${ }^{1} \mathrm{H}-\mathrm{NMR}\left(270 \mathrm{MHz}, \mathrm{CDCl}_{3}\right) \delta: 0.85(3 \mathrm{H}, \mathrm{d}$, $J=6.9 \mathrm{~Hz}), 1.03(3 \mathrm{H}, \mathrm{d}, J=6.8 \mathrm{~Hz}), 1.09-1.24(3 \mathrm{H}, \mathrm{m}), 2.54-2.65(1 \mathrm{H}$, $\mathrm{m}), 3.13(2 \mathrm{H}, \mathrm{d}, J=7.6 \mathrm{~Hz}), 3.92(1 \mathrm{H}, \mathrm{t}, J=7.6 \mathrm{~Hz}), 4.12(2 \mathrm{H}, \mathrm{q}, J=7.1 \mathrm{~Hz})$ $7.13-7.30(5 \mathrm{H}, \mathrm{m}) .{ }^{13} \mathrm{C}-\mathrm{NMR}\left(68 \mathrm{MHz}, \mathrm{CDCl}_{3}\right) \delta: 14.1,17.7,19.8,34.4$, $41.4,58.7,61.4,126.5,128.4(\times 2), 128.8(\times 2), 138.3,168.9,208.3$. FABHR-MS $m / z$ : 249.1496 (Calcd for $\mathrm{C}_{15} \mathrm{H}_{21} \mathrm{O}_{3}: 249.1485$ ).

Methyl 2-Cyano-3-phenylpropanoate (14) IR (neat) $\mathrm{cm}^{-1}:$ 2962, $2252,1751,1265,755 .{ }^{1} \mathrm{H}-\mathrm{NMR}\left(270 \mathrm{MHz}, \mathrm{CDCl}_{3}\right) \delta: 3.15(2 \mathrm{H}, \mathrm{ddd}$, $J=28.0,13.9,8.4 \mathrm{~Hz}), 3.66(1 \mathrm{H}, \mathrm{dd}, J=8.4,5.8 \mathrm{~Hz}), 3.70(3 \mathrm{H}, \mathrm{s}), 7.17-$ $7.30(5 \mathrm{H}, \mathrm{m}) .{ }^{13} \mathrm{C}-\mathrm{NMR}\left(68 \mathrm{MHz}, \mathrm{CDCl}_{3}\right) \delta: 35.8,39.6,53.5,115.9,127.7$, $128.8(\times 2), 128.9(\times 2), 135.1,165.8$. FAB-HR-MS $m / z: 188.0715($ Calcd for $\mathrm{C}_{11} \mathrm{H}_{10} \mathrm{NO}_{2}$ : 188.0712).

3-Phenyl-2-(phenylsulfonyl)propanenitrile $\quad(15)^{24)}$ IR (neat) $\mathrm{cm}^{-1}$ : 2930, 2220, 1320, $1160 .{ }^{1} \mathrm{H}-\mathrm{NMR}\left(270 \mathrm{~Hz}, \mathrm{CDCl}_{3}\right) \delta: 3.08(1 \mathrm{H}, \mathrm{dd}, J=13.6$, $11.7 \mathrm{~Hz}), 3.58(1 \mathrm{H}, \mathrm{dd}, J=13.6,3.9 \mathrm{~Hz}), 4.08(1 \mathrm{H}, \mathrm{dd}, J=11.7,3.9 \mathrm{~Hz})$ $7.21-8.06(10 \mathrm{H}, \mathrm{m}) .{ }^{13} \mathrm{C}-\mathrm{NMR}\left(68 \mathrm{MHz}, \mathrm{CDCl}_{3}\right) \delta: 32.7,59.4,113.72$, $128.2,128.6,129.1(\times 2), 129.2(\times 2), 129.7(\times 2), 130.6,133.5,135.4$, 135.5

1,3-Diphenylpropan-1-one (16) ${ }^{25)}$ IR (neat) $\mathrm{cm}^{-1}: 2352,2337,1689$, 1596. ${ }^{1} \mathrm{H}-\mathrm{NMR}\left(270 \mathrm{MHz}, \mathrm{CDCl}_{3}\right) \delta: 2.99(2 \mathrm{H}, \mathrm{t}, J=7.7 \mathrm{~Hz}), 3.23(2 \mathrm{H}, \mathrm{t}$ $J=7.7 \mathrm{~Hz}), 7.04-7.51(9 \mathrm{H}, \mathrm{m}), 7.86-7.90(2 \mathrm{H}, \mathrm{m}) .{ }^{13} \mathrm{C}-\mathrm{NMR}(68 \mathrm{MHz}$, $\left.\mathrm{CDCl}_{3}\right) \delta: 30.2,40.5,126.0,127.9(\times 2), 128.3,128.4(\times 2), 128.5(\times 2)$, $132.9(\times 2), 136.7,141.1,199.0$.

2-Methyl-1,3-diphenylpropan-1-one (17) ${ }^{26)}$ IR (neat) $\mathrm{cm}^{-1}: 2360$, 2337, 1681, 1450, 971. ${ }^{1} \mathrm{H}-\mathrm{NMR}\left(270 \mathrm{MHz}, \mathrm{CDCl}_{3}\right) \delta: 1.13(3 \mathrm{H}, \mathrm{d}$ $J=6.9 \mathrm{~Hz}), 2.62(1 \mathrm{H}, \mathrm{dd}, J=13.6,7.9 \mathrm{~Hz}), 3.10(1 \mathrm{H}, \mathrm{dd}, J=13.6,6.4 \mathrm{~Hz})$, $3.61-3.72(1 \mathrm{H}, \mathrm{m}), 7.08-7.22(5 \mathrm{H}, \mathrm{m}), 7.34-7.50(3 \mathrm{H}, \mathrm{m}), 7.83-7.86$ $(2 \mathrm{H}, \mathrm{m}) .{ }^{13} \mathrm{C}-\mathrm{NMR}\left(68 \mathrm{MHz}, \mathrm{CDCl}_{3}\right) \delta: 17.5,39.4,42.8,126.1,128.1(\times 2)$, $128.2(\times 2), 128.4(\times 2), 128.9(\times 2), 132.7,136.3,139.8,203.5$.

2,2-Dimethyl-1,3-diphenylpropan-1-one (18) ${ }^{27)}$ IR (neat) $\mathrm{cm}^{-1}: 2360$, 2329, 2673, 1457. ${ }^{1} \mathrm{H}-\mathrm{NMR}\left(270 \mathrm{MHz}, \mathrm{CDCl}_{3}\right) \delta: 1.23(6 \mathrm{H}, \mathrm{s}), 3.00(2 \mathrm{H}, \mathrm{s})$ $7.01-7.47(10 \mathrm{H}, \mathrm{m}) .{ }^{13} \mathrm{C}-\mathrm{NMR}\left(68 \mathrm{MHz}, \mathrm{CDCl}_{3}\right) \delta: 26.2(\times 2), 46.3,48.8$, $126.3,127.3(\times 2), 127.90(\times 2), 127.94,128.2,128.5,130.4,130.5,132.7$, $137.8,209.3$.

4,4-Dimethyl-1-phenylpentan-3-one (19) ${ }^{25)}$ IR (neat) $\mathrm{cm}^{-1}: 2352$ $1704,1095,694 .{ }^{1} \mathrm{H}-\mathrm{NMR}\left(270 \mathrm{MHz}, \mathrm{CDCl}_{3}\right) \delta: 1.10(9 \mathrm{H}, \mathrm{s}), 2.75-2.90$ $(4 \mathrm{H}, \mathrm{m}), 7.16-7.36(5 \mathrm{H}, \mathrm{m}) .{ }^{13} \mathrm{C}-\mathrm{NMR}\left(68 \mathrm{MHz}, \mathrm{CDCl}_{3}\right) \delta: 26.4(\times 3)$, $30.1,38.5,44.1,125.9(\times 2), 127.7,128.3(\times 2), 141.4,214.7$.

Isopropyl 3-Phenylpropanoate (20) ${ }^{28)}$ IR (neat) $\mathrm{cm}^{-1}: 3880,2337$, 1727, $1110 .{ }^{1} \mathrm{H}-\mathrm{NMR}\left(270 \mathrm{MHz}, \mathrm{CDCl}_{3}\right) \delta: 1.19(6 \mathrm{H}, \mathrm{d}, J=6.4 \mathrm{~Hz}), 2.57$ $(2 \mathrm{H}, \mathrm{t}, J=7.8 \mathrm{~Hz}), 2.93(2 \mathrm{H}, \mathrm{t}, J=7.8 \mathrm{~Hz}), 4.92-5.03(1 \mathrm{H}, \mathrm{m}), 7.17-7.36$ $(5 \mathrm{H}, \mathrm{m}) .{ }^{13} \mathrm{C}-\mathrm{NMR}\left(68 \mathrm{MHz}, \mathrm{CDCl}_{3}\right) \delta: 21.9(\times 2), 31.1,36.3,67.7,126.1$, $128.2(\times 2), 128.3(\times 2), 140.5,172.3$.

Acknowledgments This study was supported by in part by the Grant of the 21st Century COE Program, Ministry of Education, Culture, Sports, Science and Technology. The authors wish to thanks to Yamanouchi Pharmaceutical Co., Ltd. for mass spectrometry analysis and elemental analysis.

\section{References and Notes}

1) Waring A. J., "Comprehensive Organic Chemistry," ed. by Stoddart J. F., University of Sheffield, Oxford, 1979, pp. 1017-1104.

2) Sutherland I. O., "Comprehensive Organic Chemistry," ed. by Sutherland I. O., University of Liverpool, Oxford, 1979, pp. 869—956.

3) Brown J. M., "Comprehensive Organic Chemistry," ed. by Sutherland I. O., University of Liverpool, Oxford, 1979, pp. 779-814.

4) le Noble W. J., Puerta J. E., Tetrahedron Lett., 7, 1087-1090 (1966).

5) Mitsunobu O., Yamada M., Mukaiyama T., Bull. Chem. Soc. Jpn., 40, 935-939 (1967).

6) Tsunoda T., Ozaki F., Itô S., Tetrahedron Lett., 35, 5081-5082 (1994).

7) Tsunoda T., Nagaku M., Nagino C., Kawamura Y., Ozaki F., Hioki H., Itô S., Tetrahedron Lett., 36, 2531-2534 (1995).

8) Hendrickson J. B., Schwartzman S. M., Tetrahedron Lett., 16, 273 276 (1975)

9) Coburn M. D., Hayden H. H., Synthesis, 1986, 490-492 (1986).

10) Garcia B. A., Poole J. L., Gin D. Y., J. Am. Chem. Soc., 119, 75977598 (1997).

11) Takuwa T., Onishi J. Y., Matsuo J., Chem. Lett., 33, 8-9 (2004).

12) Matsuo J., Yamanaka H., Kawana A., Mukaiyama T., Chem. Lett., 32 , 392-393 (2003).

13) Yamanaka H., Matsuo J., Kawana A., Mukaiyama T., Chem. Lett., 32, 626-627 (2003).

14) Sodium enolates were prepared by adding sodium hydride in THF (Tables $1-4$ and Table 5, Entries 1-5) or sodium hexamethyldisilazanide in THF (Table 5, Entris 6-10, Tables 6 and 7) to THF solution of the corresponding carbonyl compounds.

15) House H. O., Larson J. K., Muller H. C., J. Org. Chem., 33, 961-968 (1968).

16) Elinson M. N., Feducovich S. K., Zakharenkov A. A., Ugrak B. I., Nikishin G. I., Lindeman S. V., Struchkov J. T., Tetrahedron, 51, 5035-5046 (1995).

17) Bradly R., Jeffrey S., Christpher S., Phosphorus, Sulfur and Silicon and the Related Elements, 177, 1881-1884 (2002).

18) Chuang C., Wang S., Tetrahedron, 54, 10043-10052 (1998).

19) Matheson R. C., WO200021941 (2000).

20) Palmer B. D., Lee Ho H., Johnson P., Baguley B. C., Wickham G., Wakelin L. P. G., McFadyen W. D., Denny W. A., J. Med. Chem., 33, 3008-3014 (1990).

21) Matoba K., Yamazaki T., Chem. Pharm. Bull., 31, 2955-2956 (1983).

22) Boiadjiev S. E., Lightner D. A., J. Phys. Org. Chem., 12, 751-757 (1999).

23) Sundar N., Bhat S. V., Synthetic Commun., 28, 2311-2316 (1998).

24) Fujii M., Nakamura K., Mekata H., Oka S., Ohno A., Bull. Chem. Soc. Jpn., 61, 495-500 (1988).

25) Dieter R. K., Sharma R. R., Yu H., Gore V. K., Tetrahedron, 59, 1083-1094 (2003).

26) Nudelman N. S., García G. V., J. Org. Chem., 66, 1387-1394 (2001).

27) Barluenga J., Aguilar E., Olano B., Fustero S., J. Org. Chem., 53, 1741-1744 (1988).

28) Kunishima M., Kawachi C., Morita J., Terao K., Iwasaki F., Tani S., Tetrahedron, 55, 13159-13170 (1999). 